\title{
On the Theory of Reflection of Electromagnetic Waves From the Interface Between a Compressible Magnetoplasma and a Dielectric
}

\author{
James R. Wait
}

Contribution From the Central Radio Propagation Laboratory, National Bureau of Standards, Boulder, Colo.

(Received June 24, 1964)

\begin{abstract}
It is assumed that a warm plasma may be described in terms of a continuum theory of fluid dynamies in combination with Maxwell's equations. The motions of the heavy ions are neglected but collisions with electrons are accounted for by a constant collision frequency. For these conditions, a solution is given for the reflection coefficient when a plane wave is incident obliquely onto a homogeneous half space of plasma. A d-c magnetic field is superimposed on the plasma in a direction parallel to the interface between the plasma and the dielectric. It is shown that previous solutions for a cold anisotropic plasma and a warm isotropic plasma are recovered as special cases.
\end{abstract}

\section{Introduction}

In theoretical studies of wave propagation in ionized media, it is usually assumed that the plasma is cold. This is particularly true in the vast literature devoted to ionospheric radio propagation [e.g., Ratcliffe, 1959]. In many cases this is probably well justified because the kinetic temperature of the plasma is sufficiently low. Nevertheless, it would seem to be desirable to investigate the consequences of this cold plasma assumption in some specific problems. For example, in studying the propagation of VLF radio waves, the ionosphere may often be approximated by a sharply bounded homogeneous plasma [Wait, 1962]. In this paper an explicit expression for the reflection coefficient is derived under the condition that the electroacoustic velocity is finite. In order to simplify the discussion, the superimposed $d-c$ magnetic field is taken to be parallel to the interface and transverse to the direction of propagation. In the limiting case of a cold plasma this problem reduces to one first solved explicitly by Barber and Crombie [1959].

\section{Preliminary Considerations}

The plasma medium is regarded as a one-component electron fluid. In other words, the ions are neglected in the equation of motion, yet their presence is required to neutralize the plasma. However, collisions between electrons and the neutral particles are accounted for by an energy independent collision frequency $\nu$. It is also assumed that the amplitude of the plasma and the electromagnetic oscillations are sufficiently small that linearized equations are valid [Oster, 1960]. It is further assumed that the drift velocity of the electrons is zero, so the plasma as a whole is considered stationary. The average number density of electrons is denoted $n_{0}$, which is regarded as constant in the plasma region. The mean velocity is $\vec{v}$, while their pressure deviation is $p$. The electric and magnetic fields are designated by their usual symbols $\vec{E}$ and $\vec{H}$, respectively.

A uniform magnetic field $B_{0}$ is assumed to be impressed throughout the plasma. This constant magnetic field is taken to be parallel to the $y$ axis of a suitably chosen Cartesian coordinate system $(x, y, z)$. The field quantities $\vec{E}, \vec{H}, p$, and $\vec{v}$ vary with time $t$ as $\exp (i \omega t)$ where $\omega$ is the angular frequency.

For the situation described above, the linearized equation of motion for the electrons is

$$
m n_{0}(\nu+i \omega) \vec{v}=n_{0} e\left(\vec{E}+\vec{v} \times \hat{y} B_{0}\right)-\nabla p,
$$

where $\hat{y}$ is a unit vector in the $y$-direction, and $e$ and $m$ are the charge and mass of the electron, respectively. As a consequence of assuming a scalar pressure term, Landau damping in the plasma is neglected. The linearized equation of continuity, combined with the equation of state, leads readily to

$$
u^{2} m n_{0} \nabla \cdot \vec{v}=-i \omega p,
$$

where $u$ is the velocity of sound in the electron gas. Maxwell's equations for the plasma in the absence of sources are given by

and

$$
\nabla \times \vec{E}=-i \mu_{0} \omega \vec{H}
$$

$$
\nabla \times \vec{H}=i \epsilon_{0} \omega \vec{E}+n_{0} e \vec{v}
$$


where $\mu_{0}$ and $\epsilon_{0}$ are the permeability and dielectric constant of free space, respectively.

In the problem considered in this paper, there are two distinct regions separated by a plane interface at $z=0$. The plasma region occupies the upper half space $z>0$, while a dielectric of electrical constants $\mu_{0}$ and $\epsilon_{d}$ occupies the lower half space $z<0$. Following the assumption of earlier workers [Cohen, 1962 ; Hessel et al., 1962 ; Fedorchenko, 1962], the boundary condition on the electron velocity $\vec{v}$ is that its normal component be zero at the interface $z=0$. The question whether an actual interface between a dielectric and plasma is totally effective in this regard is not answered here. However, the reader is referred to an illuminating discussion of this point by Cohen [1962]. Very recently the same boundary condition has been used by Yildiz [1963], who treated the oscillations of an isotropic compressible plasma sphere.

To simplify the problem, the obliquely incident wave from the lower half space propagates transverse to the magnetic field. In other words, the wave normal is contained in the $(x, z)$ plane and makes an angle $\theta$ with the negative $z$-axis. Without losing further generality, it is necessary to consider two distinct cases. The first and most interesting situation is when the magnetic field of the incident wave is parallel to the interface. In other words, $\vec{H}^{(i)}$ of the incident wave has only a $y$ component $H_{y}^{(i)}$. Therefore, we choose it to be of the form

$$
H_{y}^{(i)}=H_{0} e^{-u_{0} z} e^{-i \lambda x},
$$

where $u_{0}=i\left(\epsilon_{d} \mu_{0}\right)^{1 / 2} \omega \cos \theta$ and $\lambda=\left(\epsilon_{d} \mu_{0}\right)^{1 / 2} \omega \sin \theta$. For the problem as defined, it is found that the necessary equations and the boundary conditions are satisfied if the reflected wave also has only a $y$ component $H_{y}^{(r)}$. In fact, we may write

$$
H_{y}^{(r)}=R_{\|} H_{0} e^{+u_{0} z} e^{-i \lambda x},
$$

where, by definition, $R_{\|}$is the reflection coefficient.

The total magnetic field $H_{y}$ in the region $z<0$ may be written

$$
H_{y}=H_{0}\left[e^{-i k C z}+R_{\|} e^{i k C z}\right] e^{-i k S x},
$$

where $C=\cos \theta, S=\sin \theta$, and $k=\left(\epsilon_{d} \mu_{0}\right)^{1 / 2} \omega$ is the wave number in the dielectric region. The electric field components in the dielectric are obtained from Maxwell's equations. Thus,

$$
E_{z}=\frac{1}{i \epsilon_{d} \omega} \frac{\partial H_{y}}{\partial x}
$$

and

$$
E_{x}=-\frac{1}{i \epsilon_{d} \omega} \frac{\partial H_{y}}{\partial z}
$$

for the region $z<0$. The normal wave impedance $Z$, at the interface, is defined by

$$
Z=\left(\begin{array}{c}
\text { Limit } \\
z \rightarrow 0
\end{array}\right) \frac{E_{x}}{H_{y}}
$$

On combining (7) and (9), it is seen that

$$
Z=\eta C\left[\frac{1-R_{\|}}{1+R_{\|}}\right],
$$

where $\eta=k /\left(\boldsymbol{\epsilon}_{d} \omega\right)=\left(\mu_{0} / \boldsymbol{\epsilon}_{d}\right)^{1 / 2}$ is the characteristic impedance of the dielectric. Conversely, the reflection coefficient may be expressed conveniently in terms of the normal surface impedance via the relation

$$
R_{\|}=\frac{\eta C-Z}{\eta C+Z}
$$

It is apparent from the above development that the problem boils down to finding an expression for the surface impedance of the compressible magnetoplasma at the interface $z=0$. Because of the continuity of the tangential fields $E_{x}$ and $H_{y}$, this impedance is simply $Z$.

The second case alluded to above corresponds to the situation where the electric field of the incident wave is parallel to the interface and has only a $y$ component. As it turns out, this is a rather trivial case from the electromagnetic viewpoint. Neither the d-c magnetic field nor the pressure play any role. Thus, the solution for the reflection coefficient may be obtained by regarding the half space as a cold isotropic plasma. This case will not be discussed any further.

\section{Development of Equations for the Plasma}

As specified in the preceding section, the magnetic field need have only a $y$ component $H_{y}$. In what follows, the subscript $y$ on this quantity is dropped. Furthermore, because of the assumed direction of incidence $\partial / \partial y=0$. Consequently, (1) in component form may be written

and

$$
(\nu+i \omega) m n_{0} v_{x}=n_{0} e\left(E_{x}-v_{z} B_{0}\right)-\partial p / \partial x,
$$

$$
(\nu+i \omega) m n_{0} v_{z}=n_{0} e\left(E_{z}+v_{x} B_{0}\right)-\partial p / \partial z .
$$

By simple algebraic manipulation, (13) and (14) may be rewritten in the form

$$
\begin{aligned}
& v_{x}=\frac{e}{g m \alpha} E_{x}+\frac{e \omega_{c}}{m g^{2} \alpha} E_{z}-\frac{1}{g \alpha m n_{0}} \frac{\partial p}{\partial x}-\frac{\omega_{c}}{g^{2} m n_{0} \alpha} \frac{\partial p}{\partial z}, \\
& v_{z}=-\frac{e \omega_{c}}{m g^{2} \alpha} E_{x}+\frac{e}{g m \alpha} E_{z}+\frac{\omega_{c}}{g^{2} m n_{0} \alpha} \frac{\partial p}{\partial x}-\frac{1}{g \alpha m n_{0}} \frac{\partial p}{\partial z},
\end{aligned}
$$

where $\alpha=1+\left(\omega_{c} / g\right)^{2}$, where $g=\nu+i \omega$ is a convenient complex frequency parameter and $\omega_{c}=-e B_{0} / m$ is the (angular) gyrofrequency for electrons.

Maxwell's curl $\vec{H}$ equation in component form is

$$
-\frac{\partial H}{\partial z}=i \epsilon_{0} \omega E_{x}+n_{0} e v_{x},
$$


and

$$
\frac{\partial H}{\partial x}=i \epsilon_{0} \omega E_{z}+n_{0} e v_{z} .
$$

This pair may be used in conjunction with (15) and (16) to eliminate $E_{x}$ and $E_{z}$. Thus,

$$
\begin{aligned}
v_{x}=-\frac{i e \omega_{c}}{m g^{2} \omega \epsilon_{0} K \alpha}[ & {\left[\frac{\partial H}{\partial x}-\frac{i \epsilon_{0} \omega}{n_{0} e} \frac{\partial p}{\partial z}\right] } \\
& -\frac{e\left(1+\frac{\omega_{0}^{2}}{i \omega g}\right)}{m i \omega g \epsilon_{0} K \alpha}\left[\frac{\partial H}{\partial z}+\frac{i \epsilon_{0} \omega}{n_{0} e} \frac{\partial p}{\partial x}\right],
\end{aligned}
$$

and

$$
\begin{aligned}
v_{z}=-\frac{i e \omega_{c}}{m g^{2} \omega \epsilon_{0} K \alpha}[ & {\left[\frac{\partial H}{\partial z}+\frac{i \epsilon_{0} \omega}{n_{0} e} \frac{\partial p}{\partial x}\right] } \\
& +\frac{e\left(1+\frac{\omega_{0}^{2}}{i \omega g}\right)}{m i \omega g \epsilon_{0} K \alpha}\left[\frac{\partial H}{\partial x}-\frac{i \epsilon_{0} \omega}{n_{0} e} \frac{\partial p}{\partial z}\right],
\end{aligned}
$$

where $\omega_{0}^{2}=\left(n_{0} e^{2}\right) /\left(\epsilon_{0} m\right)$ is the (angular) plasma frequency,

$$
\alpha=1+\left(\omega_{c} / g\right)^{2}, K=K_{1}^{2}-K_{2}^{2}
$$

with

$$
K_{1}=\left(1+\frac{\omega_{0}^{2}}{i \omega g \alpha}\right) \text {, and } K_{2}=\frac{\omega_{0}^{2} \omega_{c}}{g^{2} \omega \alpha} .
$$

Equations (17) and (18), used in conjunction with (15) and (16), may be utilized to eliminate $v_{x}$ and $v_{z}$. From this process, we obtain

$$
E_{x}=-\frac{K_{1}}{i \epsilon_{0} \omega K} \frac{\partial H}{\partial z}-\frac{K_{2}}{\epsilon_{0} \omega K} \frac{\partial H}{\partial x}-\frac{K_{1}-K}{n_{0} e K} \frac{\partial p}{\partial x}+\frac{i K_{2}}{n_{0} e K} \frac{\partial p}{\partial z},
$$

and

$$
E_{z}=\frac{K_{1}}{i \epsilon_{0} \omega K} \frac{\partial H}{\partial x}-\frac{K_{2}}{\epsilon_{0} \omega K} \frac{\partial H}{\partial z}-\frac{K_{1}-K}{n_{0} e K} \frac{\partial p}{\partial z}-\frac{i K_{2}}{n_{0} e K} \frac{\partial p}{\partial x} .
$$

It is evident from the above that all quantities of interest are derivable from the two scalars $p$ and $H$. By inserting $v_{x}$ and $v_{z}$, as given by (19) and (20), into (2), we obtain

$$
\frac{\left(\frac{i \omega_{0}^{2}}{g \omega}\right) B_{0}}{\left(1+\frac{\omega_{0}^{2}}{i \omega g}\right)} \nabla^{2} H-\left[\nabla^{2}-\Gamma_{p}^{2}\right] p=0
$$

where

$$
\nabla^{2}=\frac{\partial^{2}}{\partial x^{2}}+\frac{\partial^{2}}{\partial z^{2}}, \text { and } \Gamma_{p}^{2}=\frac{i \omega g \alpha K}{u^{2}\left(1+\frac{\omega_{0}^{2}}{i \omega g}\right)} .
$$

In a similar manner, by working with (3), (21), and (22), we arrive at

$$
\left(\nabla^{2}-\Gamma_{e}^{2}\right) H+\frac{K_{2} \epsilon_{0} \omega}{K_{1} n_{0} \ell} \nabla^{2} p=0
$$

where

$$
\Gamma_{e}^{2}=-\frac{\mu_{0} \epsilon_{0} \omega^{2} K}{K_{1}}
$$

Equations (23) and (24) are coupled differential equations for $H$ and $p$. An important special case is when $B_{0}=0$. Then, because $K_{2}=0$, these two equations decouple. Thus,

where

$$
\left(\nabla^{2}-\gamma_{p}^{2}\right) p=0
$$

and

$$
\gamma_{p}^{2}=\frac{i \omega g}{u^{2}}\left(1+\frac{\omega_{0}^{2}}{i \omega g}\right)
$$

where

$$
\left(\nabla^{2}-\gamma_{e}^{2}\right) H=0
$$

$$
\gamma_{e}^{2}=-\mu_{0} \epsilon_{0} \omega^{2}\left(1+\frac{\omega_{0}^{2}}{i \omega g}\right) .
$$

These are the governing equations for propagation in an isotropic compressible plasma when collisions are accounted for [Wait, 1964].

Another significant special case is when the temperature of the plasma is set equal to zero. This corresponds to $\Gamma_{p} \rightarrow \infty$ whence, from (23), the pressure $p$ vanishes. Equation (24) then simplifies to

$$
\left(\nabla^{2}-\Gamma_{e}^{2}\right) H=0,
$$

where $\Gamma_{e}^{2}$ is defined exactly as in (24). This is the wave equation for transverse propagation in a cold anisotropic plasma [Wait, 1962].

The general coupled differential equations given by (23) and (24), in the case of zero collisions, are the same as the ones derived by Seshadri [1963] who treated the radiation from a magnetic line source embedded in a homogeneous compressible plasma.

\section{Solution of the Coupled Equations}

To obtain the solution of the coupled equations, it is assumed that the waves in the plasma vary according to the factor $\exp (-u z) \exp (-i \lambda x)$ where $\lambda=k$ $\sin \theta$, and $u$ is to be determined. Because $\nabla^{2}$ may be replaced by $u^{2}-\lambda^{2}$ it follows, from (23) and (24), that

$$
\left(u^{2}-\lambda^{2}-\Gamma_{e}^{2}\right)\left(u^{2}-\lambda^{2}-\Gamma_{p}^{2}\right)-\delta\left(u^{2}-\lambda^{2}\right)^{2}=0,
$$

where

$$
\delta=\frac{i \omega_{c} K_{2}}{g K_{1}}\left(1+\frac{\omega_{0}^{2}}{i \omega g}\right)^{-1}
$$

When the gyrofrequency approaches zero, the coupling factor $\delta$ vanishes, and the two solutions are $u^{2}=\lambda^{2}+\Gamma_{e}^{2}$ and $\lambda^{2}+\Gamma_{p}^{2}$. In accordance with earlier work, these may be described as electromagnetic and acoustic waves, respectively. 

form

Equation (28) is now conveniently written in the

$$
\left(u^{2}-\lambda^{2}-\hat{\Gamma}_{e}^{2}\right)\left(u^{2}-\lambda^{2}-\widehat{\Gamma}_{p}^{2}\right)=0,
$$

and

$$
\widehat{\Gamma}_{e}^{2}=v+\left(v^{2}-w\right)^{1 / 2},
$$

with

$$
\hat{\Gamma}_{p}^{2}=v-\left(v^{2}-w\right)^{1 / 2},
$$

$$
v=\frac{\Gamma_{p}^{2}+\Gamma_{e}^{2}}{2(1-\delta)} \text { and } w=\frac{\Gamma_{p}^{2} \Gamma_{e}^{2}}{1-\delta} \text {. }
$$

The sign of the radicals is chosen so that $\hat{\Gamma}_{e}^{2}$ approaches $\Gamma_{e}^{2}$ and $\hat{\Gamma}_{p}^{2}$ approaches $\Gamma_{p}^{2}$ as $\delta$ tends uniformly to zero. The solutions of (29) then lead to two kinds of waves which are described by the factors

$\exp \left(-u_{e} z\right) \exp (-i \lambda x)$ and $\exp \left(-u_{p} z\right) \exp (-i \lambda x)$,

where

$$
u_{e}=\left(\lambda^{2}+\hat{\Gamma}_{e}^{2}\right)^{1 / 2} \text { and } u_{p}=\left(\lambda^{2}+\hat{\Gamma}_{p}^{2}\right)^{1 / 2} \text {. }
$$

It is appropriate to describe these as quasi-electromagnetic and quasi-acoustic waves, respectively. The radicals in the expression for $u_{e}$ and $u_{p}$ must be chosen to satisfy radiation conditions as $z \rightarrow \infty$. In the case of a finite collision frequency $\nu$, this is equivalent to assuring that the real parts of $u_{e}$ and $u_{p}$ are positive.

\section{Derivation of the Surface Impedance}

General expressions for the magnetic field $H$ and the pressure $p$ within the plasma may be expressed in the form

$$
H=\left[f_{e} \exp \left(-u_{e} z\right)+f_{p} \exp \left(-u_{p} z\right)\right] \exp (-i \lambda x),
$$

and

$$
p=\left[g_{e} \exp \left(-u_{e} z\right)+g_{p} \exp \left(-u_{p} z\right)\right] \exp (-i \lambda x),
$$

where $f_{e}, g_{e}, f_{p}$, and $g_{p}$ are coefficients which do not depend on the coordinates. By using (24), which is valid for all $z>0$, it follows that

$$
g_{e}=\Lambda_{e} \frac{n_{0} e}{\epsilon_{0} \omega} f_{e},
$$

and

$$
g_{p}=\Lambda_{p} \frac{n_{0} e}{\epsilon_{0} \omega} f_{p}
$$

where

$$
\Lambda_{e}=\frac{\Gamma_{e}^{2}-\hat{\Gamma}_{e}^{2}}{\hat{\Gamma}_{e}^{2}} \frac{K_{1}}{K_{2}} \text { and } \Lambda_{p}=\frac{\Gamma_{e}^{2}-\hat{\Gamma}_{p}^{2}}{\hat{\Gamma}_{p}^{2}} \frac{K_{1}}{K_{2}} \text {. }
$$

Thus, the pressure is given in terms of $f_{e}$ and $f_{p}$ by

$p=\frac{n_{0} e}{\epsilon_{0} \omega}\left[f_{e} \Lambda_{e} \exp \left(-u_{e} z\right)+f_{p} \Lambda_{p} \exp \left(-u_{p} z\right)\right] \exp (-i \lambda x)$.

Using (20), (30), and (34) and the boundary condition $v_{z}=0$, we obtain

$$
\frac{f_{p}}{f_{e}}=-\frac{\left(i \omega_{c} / g\right)\left[u_{e}-\Lambda_{e} \lambda\right]-\left(1+\frac{\omega_{0}^{2}}{i \omega g}\right)\left(\lambda-\Lambda_{e} u_{e}\right)}{\left(i \omega_{c} / g\right)\left[u_{p}-\Lambda_{p} \lambda\right]-\left(1+\frac{\omega_{0}^{2}}{i \omega g}\right)\left(\lambda-\Lambda_{p} u_{p}\right)} .
$$

This means that the rigidity condition at the interface fixes the ratio of the quasi-acoustic wave to the quasi-electromagnetic wave. In the special case of a cold plasma (i.e., $u=0), \Lambda_{p} \rightarrow \infty$ and, therefore, the ratio $f_{p} / f_{e}$ vanishes. Another limiting case is when the plasma becomes isotropic (i.e., $\omega_{c}=0$ ). Then, again, $\Lambda_{p} \rightarrow \infty$ and $f_{p} / f_{e}$ become vanishingly small. However, in this case, a more meaningful ratio is $g_{p} / f_{e}$, which approaches the finite value $n_{0} e \lambda /\left(\epsilon_{0} \omega u_{p}\right)$ in accordance with the required behavior for an isotropic compressible plasma [Wait, 1964].

Using (21), (30), and (34), the following expression is obtained for the tangential electric field at the interface:

$$
\begin{aligned}
\left.E_{x}\right]_{z=0} & =\frac{1}{\epsilon_{0} \omega}\left[\frac{K_{1} u_{e}}{i K}+\frac{K_{2} i \lambda}{K}+\frac{K_{1}-K}{K} \Lambda_{e} i \lambda-\frac{K_{2}}{K} \Lambda_{e} i u_{e}\right] f_{e} \\
& +\frac{1}{\epsilon_{0} \omega}\left[\frac{K_{1} u_{p}}{i K}+\frac{K_{2} i \lambda}{K}+\frac{K_{1}-K}{K} \Lambda_{p} i \lambda-\frac{K_{2}}{K} \Lambda_{p} i u_{p}\right] f_{p} .
\end{aligned}
$$

According to (10), it is noted that

$$
\left.\left.E_{x}\right]_{z=0}=Z H_{y}\right]_{z=0}=Z\left(f_{e}+f_{p}\right) .
$$

Therefore, on equating (36) and (37), we arrive at the rather cumbersome expression for the surface impedance.

$$
\begin{aligned}
Z=\left(\frac{\lambda}{\epsilon_{0} \omega}\right) & \frac{i}{\left(1+\frac{f_{p}}{f_{e}}\right) K} \\
& \left\{\left[K_{2}-\frac{K_{1} u_{e}}{\lambda}+\left(K_{1}-K\right) \Lambda_{e}-\frac{K_{2} \Lambda_{e} u_{e}}{\lambda}\right]\right. \\
+ & \left.+\frac{f_{p}}{f_{e}}\left[K_{2}-\frac{K_{1} u_{p}}{\lambda}+\left(K_{1}-K\right) \Lambda_{p}-\frac{K_{2} \Lambda_{p} u_{p}}{\lambda}\right]\right\},
\end{aligned}
$$

where $f_{p} / f_{e}$ is given explicitly by (35).

As a partial check on the correctness of (38), we consider the case of a cold anisotropic plasma (i.e., $u=0)$. Then, since $f_{p} / f_{e} \rightarrow 0$ and $\boldsymbol{\Lambda}_{e} \rightarrow 0$, it immediately follows that $Z \rightarrow Z_{c}$ where

$$
Z_{c}=\frac{i}{\epsilon_{0} \omega K}\left(K_{2} \lambda-K_{1} u_{e}\right) \text { with } u_{e}=\left(\lambda^{2}+\Gamma_{e}^{2}\right)^{\frac{1}{2}},
$$


which is in agreement with a result derived originally by Barber and Crombie [1959]. Another special case is a warm isotropic plasma (i.e., $\omega_{c}=0$ ). Then, $\delta=0, \quad \hat{\Gamma}_{e} \rightarrow \Gamma_{e}, \quad \hat{\Gamma}_{p} \rightarrow \Gamma_{p}, \quad K_{2} \rightarrow 0, \quad$ and $K / K_{1} \rightarrow\left[1+\omega_{0}^{2} /\right.$ $(i \omega g)]=\epsilon / \epsilon_{0}$ where $\epsilon$ is the dielectric constant of the isotropic plasma. In this limiting case, $\left(f_{p} / f_{e}\right) \rightarrow 0$ and $\Lambda_{p} \rightarrow \infty$ but in such a way that $\left(\Lambda_{p} f_{p} / f_{e}\right) \rightarrow \lambda / u_{p}$. $A$ s a result of these considerations, $Z \rightarrow Z_{i}$ where

$$
Z_{i}=\frac{u_{e}}{i \epsilon \omega}\left[1-\frac{\lambda^{2}}{u_{p} u_{e}} \frac{i \omega_{0}^{2}}{g \omega}\right]
$$

where

$$
u_{e}=\left(\lambda^{2}+\gamma_{e}^{2}\right)^{\frac{1}{2}} \text { and } u_{p}=\left(\lambda^{2}+\gamma_{p}^{2}\right)^{\frac{1}{2}}
$$

with

and

$$
\gamma_{p}^{2}=\frac{i \omega g}{u^{2}}\left(1+\frac{\omega_{0}^{2}}{i \omega g}\right)=-\frac{\omega^{2}}{u^{2}}\left(1+\frac{\nu}{i \omega}\right) \frac{\epsilon}{\epsilon_{0}}
$$

$$
\gamma_{e}^{2}=-\epsilon_{0} \mu_{0} \omega^{2}\left(1+\frac{\omega_{0}^{2}}{i \omega g}\right)=-\epsilon \mu_{0} \omega^{2}
$$

The above result for $Z_{i}$ is in agreement with an independent derivation for an isotropic warm plasma [Wait, 1964]. It is immediately evident that the bracketed term in (39) may be replaced by unity if $u / c<<1$, which is the condition for the validity of the cold plasma assumption.

\section{Concluding Remarks}

An explicit expression for the reflection coefficient for a compressible magnetoplasma has been given in this paper. It is shown that it reduces to the well- known result for a cold magnetoplasma when the ratio of the acoustic velocity $u$ is small compared with the velocity $c$ of electromagnetic waves in free space. The general expression also reduces to a previously derived result for reflection from an isotropic compressible half space of plasma.

\section{References}

Barber, N. F., and D. D. Crombie (1959), VLF reflections from the ionosphere in the presence of a transverse magnetic field, J. Atmos. Terrest. Phys. 16, 37.

Cohen, M. H. (1962), Radiation in a plasma, Phys. Rev. 126, 389-404.

Fedorchenko, A. M. (May, 1962), Conversion of a transverse electromagnetic wave into a longitudinal wave at a dielectric-plasma boundary, Soviet Physics-Tech. Physics. (Zh. Tekh. Fiz.) 32, 589-592.

Hessel, A., N. Marcuvitz, and J. Shmoys (Jan. 1962), Scattering and guided waves at an interface between air and a compressible plasma, IRE Trans. AP-10, 48-54.

Oster. L. (Jan. 1960), Linearized theory of plasma oscillations, Rev. Mod. Phys. 32, 141-168.

Ratcliffe, J. A. (1959), Magneto-ionic theory and its applications to the ionosphere (Cambridge Univ. Press, Cambridge).

Seshadri, S. R. (Jan. 1963), Excitation of plasma waves in an unbounded homogeneous plasma by a line source, IEEE Trans. M TT- $-11,39-50$.

Wait, J. R. (1962), Electromagnetic waves in stratified media (Pergamon Press, Oxford and Macmillan Co., New York).

Wait, J. R. (Sept. 1964), Radiation from sources in compressible plasma media, Can. J. Phys. 42.

Yildiz, A. (Dec. 1963), Scattering of plane plasma waves from a plasma sphere, Il Nuovo Cimento, Serie X, 30, 11821207.

(Paper 68D11-419) 